\title{
Viewing Hybrid Systems as Products of Control Systems and Automata
}

\author{
R. L. Grossman and R. G. Larson \\ University of Illinois at Chicago
}

Feburary, 1992

\begin{abstract}
The purpose of this note is to show how hybrid systems may be modeled as products of nonlinear control systems and finite state automata. By a hybrid system, we mean a network of consisting of continuous, nonlinear control system connected to discrete, finite state automata. Our point of view is that the automata switches between the control systems, and that this switching is a function of the discrete input symbols or letters that it receives. We show how a nonlinear control system may be viewed as a pair consisting of a bialgebra of operators coding the dynamics, and an algebra of observations coding the state space. We also show that a finite automata has a similar representation. A hybrid system is then modeled by taking suitable products of the bialgebras coding the dynamics and the observation algebras coding the state spaces.
\end{abstract}

\section{Acknowledgments}

Robert Grossman's research was supported in part by NASA grant NAG2513, NSF grant DMS-9101089, and the Laboratory for Advanced Computing.

\section{Status}

Proceedings of the 31st IEEE Conference on Decision and Control, IEEE Press, 1992, to appear.

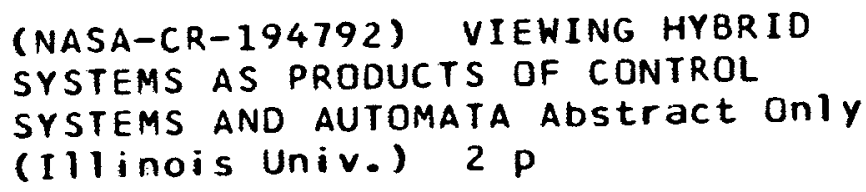

N94-23300

Unclas 


\section{References}

[1] P.E. Crouch and R. L. Grossman, "The Explicit Computation of Integration Algorithms and First Integrals for Ordinary Differential Equations With Polynomial Coefficients Using Trees," Proceedings of the 1992 International Symposium on Symbolic and Algebraic Computation, ACM Press, pp. 89-94.

[2] P.E. Crouch, R. Grossman, and Y. Yan, "On the numeric integration of dynamic attitude equations," Proceedings of the 31st IEEE Conference on Decision and Control, IEEE Press, 1992.

[3] R. Grossman and S. Mehta, and X. Qin, "Path planning by querying persistent stores of trajectory segments," September, 1992, $6 \mathrm{TEX}$ pages.

[4] R. L. Grossman, D. Likfa, and X. Qin, "An object manager utilizing hierarchical storage," Twelfth IEEE Symposium on Mass Storage Systems, IEEE Press, Los Alamites, 1993, to appear.

[5] A. Baden, L. Cormell, C. T. Day, R. Grossman, P. Leibold, D. Lifka, D. Liu, S. Loken, E. Lusk, J. F. MacFarlane, E. May, U. Nixdorf, L. E. Price, X. Qin, B. Scipioni, and T. Song, "Database Computing in HEP-Progress Report," Computing in High Energy Physics 1992, to appear.

[6] R. L. Grossman and R. G. Larson, "Viewing hybrid systems as products of control systems and automata," Proceedings of the 31st IEEE Conference on Decision and Control, IEEE Press, 1992, pp. 2953-2955. 